\title{
Hip flexor muscle fatigue in patients with symptomatic femoroacetabular impingement
}

\author{
N. C. Casartelli • M. Leunig • J. F. Item-Glatthorn • \\ R. Lepers $\cdot$ N. A. Maffiuletti
}

Received: 22 August 2011 /Accepted: 30 September 2011 /Published online: 26 October 2011

(C) Springer-Verlag 2011

\begin{abstract}
Purpose Patients with symptomatic femoroacetabular impingement (FAI) have considerable hip muscle weakness, in particular, hip flexion and hip adduction. In addition, they experience disabilities while performing prolonged dynamic tasks. It was therefore postulated that, besides hip flexor muscle weakness, patients with symptomatic FAI would show greater hip flexor fatigue compared with healthy controls.

Methods Hip flexor fatigue was evaluated in two different experiments. Fifteen patients with symptomatic FAI and 15 age-matched healthy controls were tested in each experiment. In the first one, changes in hip flexor torque fluctuations and electromyographic (EMG) activity were measured during a sustained submaximal isometric contraction. In the second experiment, hip flexor torque decline was measured during a series of 20 maximal dynamic contractions.

Results Patients with FAI exhibited hip flexor weakness under both isometric $(P=0.02)$ and isokinetic conditions $(P=0.03)$. Fatigue-induced changes in isometric hip flexor torque fluctuations, EMG root mean square and median frequency did not differ significantly between patients and
\end{abstract}

N. C. Casartelli $(\triangle) \cdot$ J. F. Item-Glatthorn · N. A. Maffiuletti Neuromuscular Research Laboratory, Schulthess Clinic, Lengghalde 2,

8008 Zurich, Switzerland

e-mail: nicola.casartelli@kws.ch

M. Leunig

Hip Service, Schulthess Clinic,

Zurich, Switzerland

R. Lepers

Inserm U887, Faculty of Sport Science, University of Burgundy,

Dijon, France controls $(P>0.05)$. Similarly, isokinetic hip flexor torque decline was comparable in patients with FAI and controls $(P>0.05)$.

Conclusions None of the hip flexor fatigue outcomes considered here differed between patients with symptomatic FAI and controls. Therefore, the disabilities that patients experience while performing prolonged dynamic tasks do not seem to be caused by exaggerated hip flexor muscle fatigue.

\section{Introduction}

Femoroacetabular impingement (FAI) has been proposed as a pathomechanical mechanism that may instigate functional disability and early development of hip osteoarthritis in young and active adults [11, 13, 14]. Patients with symptomatic FAI have considerable hip muscle weakness, that is, they present an impaired ability to develop maximal hip muscle strength compared to healthy controls [7]. Such FAI-related hip muscle weakness might cause functional disabilities during short moderate-to-high intensity dynamic tasks, such as jumping and one-leg stepping down [1]. Nevertheless, patients with symptomatic FAI also experience hip joint pain and disability while performing dynamic tasks for prolonged periods of time. For example, Clohisy et al. [8] reported that walking limitations of patients with FAI progressively worsen as walking time increases. Similarly, Philippon et al. [20] observed that almost all patients with FAI (92\%) could not practice their sport activities (e.g., running, tennis, football) as long as desired. For this reason, it is important to verify if, besides hip flexor muscle weakness, FAI may lead to impaired fatigue resistance, which is defined as the muscle's inability to maintain an expected force [2]. 
Therefore, the aim of this study was to compare hip flexor fatigue between patients with symptomatic FAI and agematched healthy controls. Muscle fatigue was investigated in submaximal static and maximal dynamic conditions, so as to imitate situations that patients could encounter during daily and sport activities. It was postulated that patients would demonstrate greater hip flexor muscle fatigue compared to controls.

\section{Materials and methods}

\section{Subjects}

Patients with symptomatic FAI and healthy control subjects matched for gender, age and body mass volunteered to participate in the study. FAI diagnosis was carried out by the same senior surgeon according to clinical and medical imaging evaluation. Approximately one-third of patients had bilateral FAI diagnosis. All patients were participating in recreational physical activities (three times a week for at least $30 \mathrm{~min}$, on average) before the onset of symptoms. Control subjects were asymptomatic and had no history of hip joint pain. Their average physical activity level was comparable to that of patients. For both groups, subjects presenting any disorder to the lower extremities (excepting hip impingement in the FAI group) that would have negatively influenced muscle fatigue evaluation were not included in the study. The study was conducted according to the Helsinki declaration, and the protocol was approved by the local Ethics Committee. All the subjects signed a written informed consent before participating in the study.

Experimental setup and procedures

Hip flexor fatigue was evaluated by comparing muscle fatigue outcomes between patients with symptomatic FAI and controls (between-subject comparison). The symptomatic (unilateral FAI) or the most symptomatic hip (bilateral FAI) was tested in patients, while controls had the respective hip evaluated, according to lower limb dominance. Hip flexor fatigue was evaluated in two separate experiments. In the first one (Experiment I), submaximal isometric fatigue was assessed in 15 patients and 15 controls (Table 1) as the changes in hip flexor torque fluctuations [18] and electromyographic (EMG) activity [4] during the course of a sustained submaximal contraction. In the second experiment (Experiment II), maximal isokinetic fatigue was evaluated in 15 patients and 15 controls (Table 1) as the decline in concentric hip flexor torque during a series of consecutive maximal contractions [6]. Ten patients and their respective controls completed both experiments in the same test session, separated by approximately ten minutes.

Hip flexor torque was measured with an isokinetic dynamometer (Biodex Medical Systems, New York, USA). Subjects laid supine on the dynamometer chair with the backrest inclined $15^{\circ}$ with respect to the horizontal, and the dynamometer rotation axis was aligned to the hip rotation centre (greater trochanter) $[7,10]$. The ipsilateral thigh was strapped to the dynamometer pad, approximately $5 \mathrm{~cm}$ proximal to the lateral femoral condyle. Pelvis and trunk were secured to the dynamometer chair with straps. Prior to fatigue evaluation, the mass of the tested limb was measured to correct for gravity.

\section{Experiment I: Submaximal isometric fatigue}

Protocol The dynamometer lever arm was fixed at $45^{\circ}$ of hip flexion [7, 10]. First, hip flexor maximal voluntary contraction (MVC) torque was determined using standard procedures [15]. After two to three submaximal contractions, subjects were asked to complete three to four MVC trials of three to four seconds, without any concern for the rate of force development. Standardised verbal encouragement was consistently provided by the investigators. Rest interval between MVC trials was 60 seconds. After five minutes, subjects performed two or three familiarisation trials by flexing the hip for five to six seconds at 20 $25 \%$ of MVC, and then completed the fatigue protocol. A computer screen that displayed the target $(50 \%$ of MVC) and the actual hip flexion torque (Fig. 1a) was consistently provided to the subjects for visual feedback.

Subjects were instructed to progressively contract their hip flexor muscles up to the target torque, and to

Table 1 Participant characteristics by experiment and group

\begin{tabular}{|c|c|c|c|c|c|c|c|}
\hline Experiment & Group & $N$ & Age (years) & Weight (kg) & Height $(\mathrm{cm})$ & BMI $\left(\mathrm{kg} \cdot \mathrm{m}^{-2}\right)$ & FAI type (cam/pincer/combined) \\
\hline I & $\begin{array}{l}\text { FAI } \\
\text { Controls }\end{array}$ & $\begin{array}{l}15(9+9) \\
15(9+9)\end{array}$ & $\begin{array}{l}31 \pm 10 \\
31 \pm 9\end{array}$ & $\begin{array}{l}72 \pm 12 \\
70 \pm 12\end{array}$ & $\begin{array}{l}175 \pm 8 \\
171 \pm 5\end{array}$ & $\begin{array}{l}23.5 \pm 3.5 \\
23.8 \pm 3.7\end{array}$ & $5 / 3 / 7$ \\
\hline II & $\begin{array}{l}\text { FAI } \\
\text { Controls }\end{array}$ & $\begin{array}{l}15(9+9) \\
15(9+9)\end{array}$ & $\begin{array}{l}32 \pm 10 \\
33 \pm 10\end{array}$ & $\begin{array}{l}70 \pm 11 \\
69 \pm 12\end{array}$ & $\begin{array}{l}174 \pm 9 \\
170 \pm 5\end{array}$ & $\begin{array}{l}22.9 \pm 3.6 \\
23.9 \pm 3.5\end{array}$ & $5 / 3 / 7$ \\
\hline
\end{tabular}

FAI femoroacetabular impingement, $B M I$ body mass index

Mean values \pm standard deviation (SD) 

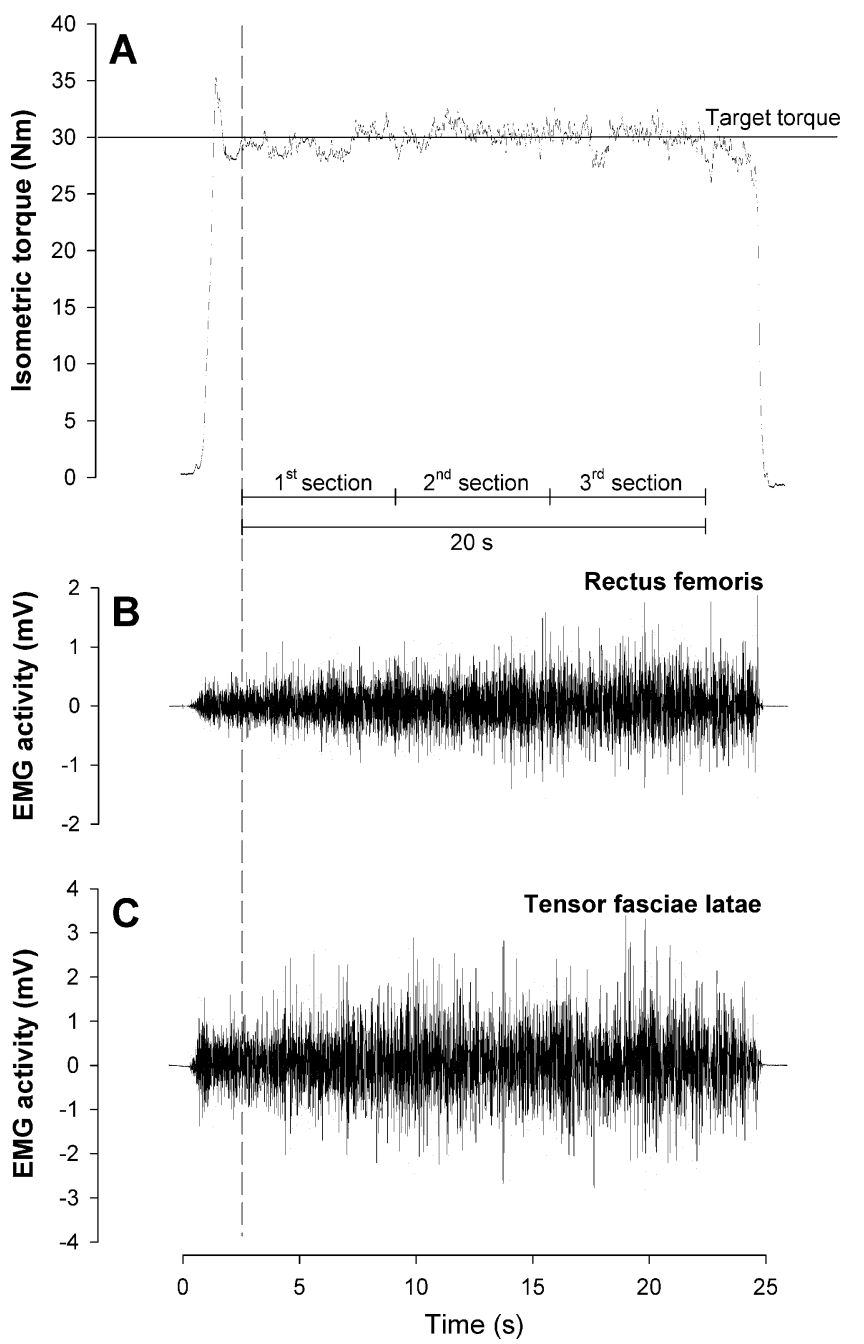

Fig. 1 Experimental traces of a sustained (25 s) isometric submaximal hip flexion in one representative subject. The different panels show the target (50\% of MVC) and the actual hip flexion torques (a), rectus femoris EMG activity (b), and tensor fasciae latae EMG activity (c). The vertical long-dashed line defines the start of the 20-s time interval considered for analysis, which was subdivided in three time sections of equal duration

maintain it for 25 seconds. During MVC and fatigue trials, torque and EMG activity of rectus femoris and tensor fasciae latae muscles were continuously recorded (Fig. 1b, c) using the Biopac system (MP150, Biopac System Inc., Goleta, CA, USA).

EMG activity Pairs of silver-chloride surface electrodes (inter-electrode distance of $25 \mathrm{~mm}$ ) were positioned on the rectus femoris and tensor fasciae latae muscles according to standard recommendations [12]. Low resistance between electrodes was achieved with light abrasion of the skin and cleaning with alcohol. The ground electrode was positioned over the ipsilateral patella. EMG signals were amplified with a bandwidth frequency ranging from $10 \mathrm{~Hz}$ to $500 \mathrm{~Hz}$, and digitised online at a sampling frequency of $2 \mathrm{kHz}$.
Data analysis Only the highest isometric MVC torque (normalised to body weight) was retained. For the fatigue test, we considered a 20 -second time interval starting from the point where actual torque stabilised around the target torque (Fig. 1). The 20-second period was further subdivided in three time sections of equal duration $(6.67 \mathrm{~s})$. Average hip flexion torque fluctuations were calculated for each time section as (coefficient of variation $=100 \mathrm{x}$ [standard deviation of torque / mean torque]). In addition, average EMG root mean square (RMS) and median frequency (MDF) of rectus femoris and tensor fasciae latae muscles were calculated for each time section. EMG data were subsequently normalised as a percentage of the respective MVC values [4] previously obtained. Analyses were performed with Acqknowledge software (Biopac System Inc., Goleta, CA, USA).

\section{Experiment II: Maximal isokinetic fatigue}

Protocol The lever arm of the isokinetic dynamometer was set to move passively at an angular velocity of $70^{\circ} \cdot \mathrm{s}^{-1}$, from $10^{\circ}$ to $80^{\circ}$ of hip flexion (range of motion, $70^{\circ}$ ). Subjects were instructed to maximally flex the hip as the lever arm moved from $10^{\circ}$ to $80^{\circ}$ of flexion, and to rest as the lever arm moved from $80^{\circ}$ to $10^{\circ}$ of flexion. After three to four submaximal contractions, subjects completed 20 consecutive isokinetic maximal contractions of the hip flexors.

Data analysis Weight-normalised isokinetic torque produced at $45^{\circ}$ of hip flexion (constant angle torque) was calculated for each of the 20 contractions. Since maximal hip flexor torque was generally observed at contraction number two, data of contraction number one were systematically excluded from the analyses and all torque values were expressed as a percentage of contraction number two [22]. Rate of torque decline was then calculated as the difference between "initial" torque (mean of contraction numbers two to four) and "final" torque (mean of contraction numbers 18 to 20 ), and further divided by the effective duration of the fatigue protocol (19 s) [16].

\section{Statistics}

Differences between patients and controls in isometric MVC torque (Experiment I) and maximal isokinetic torque (Experiment II, contraction number two) of hip flexors were assessed with paired $t$-tests, as the two groups of subjects were treated as matched pairs [5]. Two-way ANOVAs (time $\mathrm{x}$ group) with repeated measures were used to assess differences in hip flexor fatigue outcomes between patients and controls. Bonferroni post-hoc analyses were further used to detect differences between pairs of means. Significance level was 
set at $P<0.05$. Statistical analyses were performed with PASW Statistics 18.0 (SPSS Inc., Chicago, IL, USA).

\section{Results}

Experiment I: Submaximal isometric fatigue

Isometric MVC torque of hip flexors was significantly lower $(-21 \%)$ in patients than in controls $(0.96 \pm 0.46$ vs. $\left.1.21 \pm 0.38 \mathrm{~N} \cdot \mathrm{m} \cdot \mathrm{kg}^{-1} ; P=0.02\right)$. Concerning hip flexion torque fluctuations, no main effects of group and interaction were found $(P=0.53)$, while time effect was significant $(P=$ $0.03)$. Torque fluctuations significantly increased from the first to the third time section in both groups of subjects $(P=$ 0.03; Fig. 2a). Concerning EMG activity, no group effect and interaction were observed for RMS and MDF of both rectus femoris and tensor fasciae latae muscles $(P>0.05)$, whereas a significant effect of time was observed for EMG RMS and MDF of both muscles $(P<0.001)$. Post-hoc
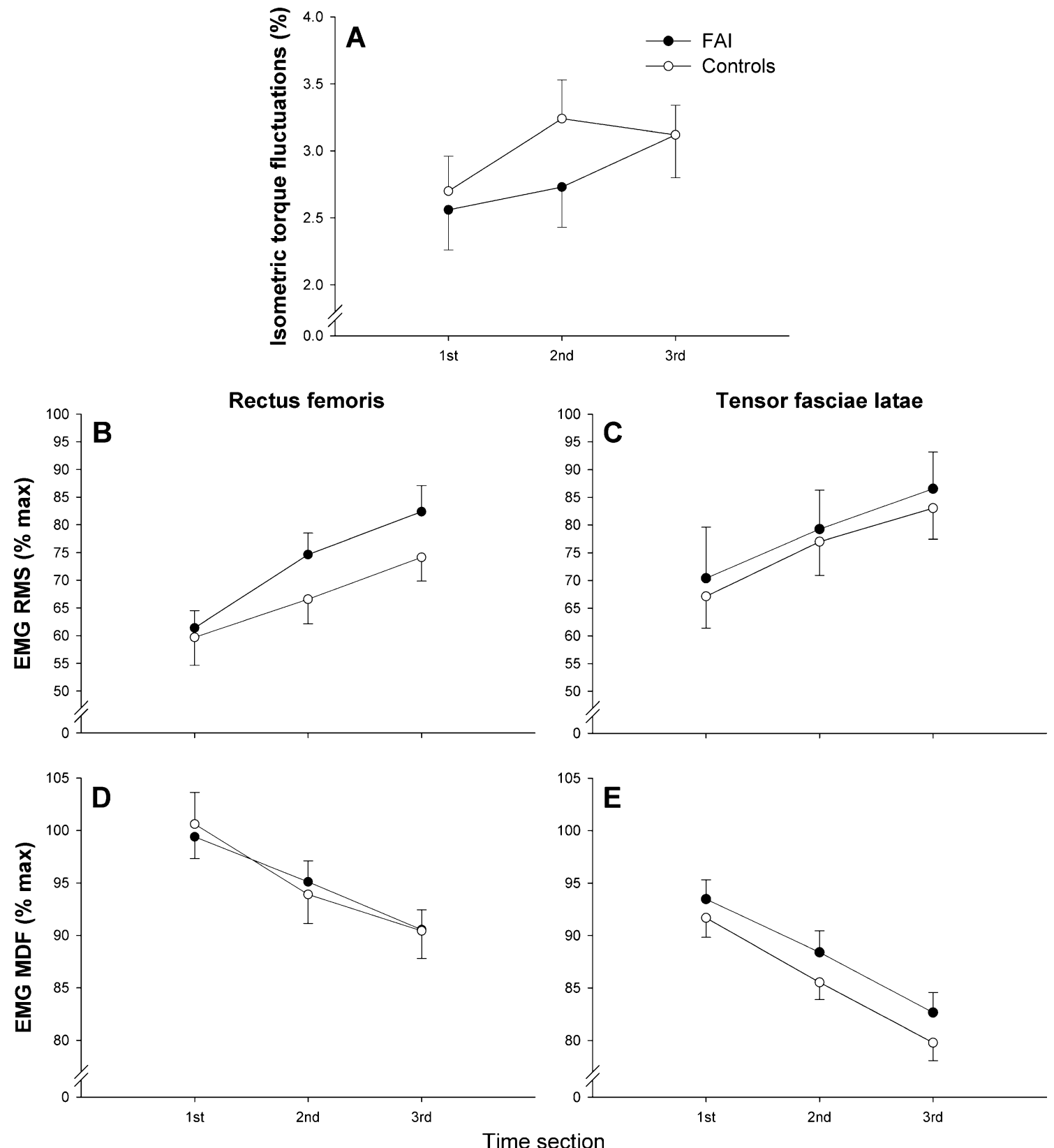

Fig. 2 Submaximal isometric hip flexor fatigue in patients with FAI $(N=15)$ and healthy controls $(N=15)$. The different panels show fatigue-related changes in hip flexor torque fluctuations (a), rectus femoris EMG RMS (b), tensor fasciae latae EMG RMS (c), rectus femoris EMG MDF (d), and tensor fasciae latae EMG MDF (e). Circles and error bars represent means and standard errors of the means. FAI femoroacetabular impingement 
analyses showed that EMG RMS and MDF significantly increased (Fig. 2b, c) and decreased (Fig. 2d, e), respectively, from one to the subsequent time section for both rectus femoris and tensor fasciae latae muscles $(P<0.01)$.

Experiment II: Maximal isokinetic fatigue

The maximal isokinetic torque of hip flexors was significantly lower $(-16 \%)$ in patients than in controls $\left(0.97 \pm 0.38\right.$ vs. $\left.1.16 \pm 0.36 \mathrm{~N} \cdot \mathrm{m} \cdot \mathrm{kg}^{-1} ; P=0.03\right)$. Isokinetic torque significantly decreased during the course of the fatigue protocol $(P<0.001$; Fig. 3$)$, but no main effect of group $(P=$ $0.80)$ and interaction $(P=0.79)$ were noted. The rate of torque decline did not differ between patients and controls $(0.017 \pm$ 0.011 vs. $0.020 \pm 0.009 \mathrm{~N} \cdot \mathrm{m} \cdot \mathrm{kg}^{-1} \cdot \mathrm{s}^{-1} ; P=0.15$ ).

\section{Discussion}

In contrast to our initial hypothesis, none of the hip flexor fatigue outcomes considered in this study differed significantly between patients with symptomatic FAI and controls, either during a sustained isometric submaximal contraction, or during a series of consecutive isokinetic maximal contractions.

Assessment of hip flexor fatigue could be of particular interest in patients with FAI, since they usually report subjective disabilities during prolonged walking [8] and sport activities such as running and tennis [20]. In this study, two distinct protocols were implemented to induce hip flexor fatigue, so as to reproduce different situations that patients could encounter during daily and sport activities. More specifically, we manipulated the main contraction characteristics, namely, the type (static vs.

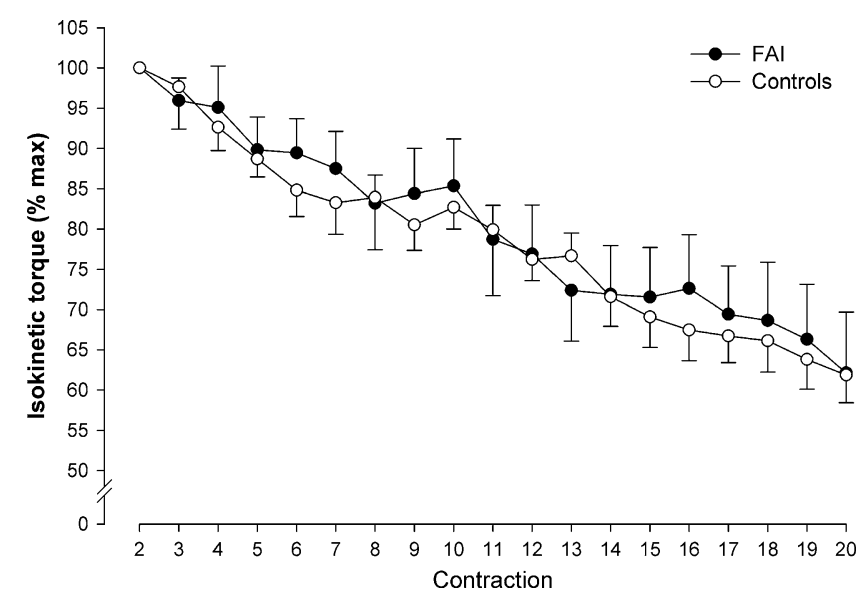

Fig. 3 Maximal isokinetic hip flexor fatigue in patients with FAI $(N=$ $15)$ and healthy controls $(N=15)$. Circles and error bars represent means and standard errors of the means. FAI femoroacetabular impingement dynamic), the intensity (submaximal vs. maximal) and the timing (sustained vs. intermittent) with the objective to increase the probability to detect a difference in hip flexor fatigue between patients and controls. To our knowledge, this combined characterisation of hip flexor muscle fatigue using sustained submaximal isometric contractions and intermittent maximal isokinetic contractions has not been previously implemented in any patient or healthy populations. We deliberately evaluated muscle fatigue of hip flexors only, as hip flexion is one of the most impaired hip movements for patients with symptomatic FAI according to pain [3], range of motion [20], and muscle strength [7]. The lack of EMG recordings from the iliopsoas muscle represents a limitation of our study, since the iliopsoas is a major hip flexor. However, this would have required the use of indwelling fine-wire electrodes. In order to avoid such an invasive approach with our patients, we only recorded surface EMG activity from the two main superficial hip flexor muscles (rectus femoris and tensor fasciae latae).

Although muscle fatigue is an important functional parameter for physical, work and daily activities, it has been rarely investigated using objective measures in orthopaedic patients. Contrary to all expectations, quadriceps muscle fatigue has been found to be less for the involved compared to the uninvolved side after anterior cruciate ligament reconstruction [17, 22]. Indeed, the operated quadriceps exhibited a lower rate of torque and EMG MDF decline during a 30-second sustained maximal isometric contraction [17], as well as a lower rate of torque decline during a series of submaximal $(20 \%$ of MVC) isometric contractions evoked by electrical stimulation [22]. Nevertheless, the patients evaluated in these particular studies had considerable quadriceps weakness (41-60\%) that probably invalidated, at least in part, fatigue comparisons (too disparate absolute torque levels were used for the involved vs. uninvolved quadriceps). It is actually difficult to compare the above-reported fatigue data with our study findings, due to considerable differences in patient populations, investigated muscles, fatigue protocols, and magnitude of muscle weakness. Nevertheless, it is certainly possible to conclude that hip flexor muscle weakness was not associated with reduced muscle fatigue in our group of FAI patients, contrary to the quadriceps studies reported above.

According to our results, subjective disabilities experienced by patients with FAI while performing dynamic tasks such as walking and running for prolonged periods of time do not seem to be caused by exaggerated hip flexor muscle fatigue. This is probably due to the dichotomy between subjective fatigue, which is usually described as an enduring, subjective sensation of generalised tiredness or lack of 
energy, and objectively-assessed fatigue, which can be further differentiated into physical (global) fatigue and muscle fatigue. Nevertheless, it is not excluded that the significant hip flexor weakness observed here $(21 \%$ and $16 \%$ for maximal isometric and isokinetic contractions, respectively) could prematurely reduce the ability of patients to develop the required muscle force during prolonged functional tasks. We cannot exclude the possibility that, if our patients and controls were compared using contractions of equal (absolute) force, then the weaker muscles of patients with FAI would have worked at a higher percentage of their maximal capacity, and as a consequence, they might have fatigued earlier and/or to a greater extent [9]. Under these conditions, FAI-related hip flexor muscle weakness could eventually induce greater hip flexor fatigue during sustained functional tasks, which might in turn limit walking ability and endurance, as recently demonstrated for neurological patients [21]. In addition, since hip flexors provide the main contribution to forward motion during early and middle swing of running [19], it can be postulated that inadequate hip flexor function can also affect running performance (including its duration) in patients with FAI.

Our hip flexor muscle weakness findings confirm recent results obtained in isometric conditions [7], and further extend these findings to concentric dynamic contractions, such as those commonly encountered in everyday life (e.g., stair climbing, walking uphill). The physiological mechanisms underlying such FAI-related muscle weakness still remain to be identified. Future research should further investigate the extent of subjective and objective physical fatigue associated with sustained functional tasks in patients with FAI, together with the motivational and physiological mechanisms underlying fatigue. In addition, since the effects of preoperative hip strengthening programs on the clinical outcomes of patients with symptomatic FAI seem worthwhile to investigate $[7,23]$, hip strengthening exercises should not be aimed at reducing hip flexor fatigability but rather to restore maximal hip flexor muscle strength to healthy control levels.

Acknowledgments The authors would like to thank Severin Staehli for his contribution in data collection.

Conflict of interest The authors declare that they have no conflict of interest.

\section{References}

1. Austin AB, Souza RB, Meyer JL, Powers CM (2008) Identification of abnormal hip motion associated with acetabular labral pathology. J Orthop Sports Phys Ther 38(9):558-565. doi:10.2519/jospt.2008.2790

2. Barry BK, Enoka RM (2007) The neurobiology of muscle fatigue: 15 years later. Integr Comp Biol 47(4):465-473. doi:10.1093/icb/ icm047

3. Beaulé PE, Allen DJ, Clohisy JC, Schoenecker P, Leunig M (2009) The young adult with hip impingement: deciding on the optimal intervention. J Bone Joint Surg Am 91(1):210 221

4. Bilodeau M, Schindler-Ivens S, Williams DM, Chandran R, Sharma SS (2003) EMG frequency content changes with increasing force and during fatigue in the quadriceps femoris muscle of men and women. J Electromyogr Kinesiol 13 (1):83-92

5. Bland JM, Altman DG (1994) Matching. BMJ 309 (6962): 1128

6. Bosquet L, Maquet D, Forthomme B, Nowak N, Lehance C, Croisier JL (2010) Effect of the lengthening of the protocol on the reliability of muscle fatigue indicators. Int J Sports Med 31(2):8288. doi:10.1055/s-0029-1243168

7. Casartelli NC, Maffiuletti NA, Item-Glatthorn JF, Staehli S, Bizzini M, Impellizzeri FM, Leunig M (2011) Hip muscle weakness in patients with symptomatic femoroacetabular impingement. Osteoarthritis Cartilage 19(7):816-821. doi:10.1016/ j.joca.2011.04.001

8. Clohisy JC, Knaus ER, Hunt DM, Lesher JM, Harris-Hayes M, Prather H (2009) Clinical presentation of patients with symptomatic anterior hip impingement. Clin Orthop Relat Res 467(3):638644. doi:10.1007/s11999-008-0680-y

9. Enoka RM, Stuart DG (1992) Neurobiology of muscle fatigue. J Appl Physiol 72(5):1631-1648

10. Frost KL, Bertocci GE, Wassinger CA, Munin MC, Burdett RG, Fitzgerald SG (2006) Isometric performance following total hip arthroplasty and rehabilitation. J Rehabil Res Dev 43 (4):435-444

11. Ganz R, Parvizi J, Beck M, Leunig M, Nötzli H, Siebenrock KA (2003) Femoroacetabular impingement: a cause for osteoarthritis of the hip. Clin Orthop Relat Res 417:112-120. doi:10.1097/01. blo.0000096804.78689.c2

12. Hermens HJ, Freriks B, Disselhorst-Klug C, Rau G (2000) Development of recommendations for SEMG sensors and sensor placement procedures. J Electromyogr Kinesiol 10 (5):361-374

13. Imam S, Khanduja V (2011) Current concepts in the diagnosis and management of femoroacetabular impingement. Int Orthop 35 (10):1427-1435. doi:10.1007/s00264-011-1278-7

14. Leunig M, Beaulé PE, Ganz R (2009) The concept of femoroacetabular impingement: current status and future perspectives. Clin Orthop Relat Res 467(3):616-622. doi:10.1007/s11999-008-0646-0

15. Maffiuletti NA (2010) Assessment of hip and knee muscle function in orthopaedic practice and research. J Bone Joint Surg Am 92:220-229

16. Maffiuletti NA, Bizzini M, Desbrosses K, Babault N, Munzinger U (2007) Reliability of knee extension and flexion measurements using the Con-Trex isokinetic dynamometer. Clin Physiol Funct Imaging 27(6):346-353. doi:10.1111/j.1475-097X.2007.00758.x

17. McHugh MP, Tyler TF, Nicholas SJ, Browne MG, Gleim GW (2001) Electromyographic analysis of quadriceps fatigue after anterior cruciate ligament reconstruction. J Orthop Sports Phys Ther 31(1):25-32

18. Missenard O, Mottet D, Perrey S (2009) Factors responsible for force steadiness impairment with fatigue. Muscle Nerve 40 (6):1019-1032. doi:10.1002/mus.21331

19. Montgomery WH III, Pink M, Perry J (1994) Electromyographic analysis of hip and knee musculature during running. Am J Sports Med 22(2):272-278 
20. Philippon MJ, Maxwell RB, Johnston TL, Schenker M, Briggs KK (2007) Clinical presentation of femoroacetabular impingement. Knee Surg Sports Traumatol Arthrosc 15(8):1041-1047. doi:10.1007/s00167-007-0348-2

21. Ramdharry GM, Day BL, Reilly MM, Marsden JF (2009) Hip flexor fatigue limits walking in Charcot-Marie-Tooth disease. Muscle Nerve 40(1):103-111. doi:10.1002/mus.21264
22. Snyder-Mackler L, Binder-Macleod SA, Williams PR (1993) Fatigability of human quadriceps femoris muscle following anterior cruciate ligament reconstruction. Med Sci Sports Exerc 25(7):783-789

23. Yazbek PM, Ovanessian V, Martin RL, Fukuda TY (2011) Nonsurgical treatment of acetabular labrum tears: a case series. J Orthop Sports Phys Ther 41(5):346-353. doi:10.2519/jospt.2011.3225 